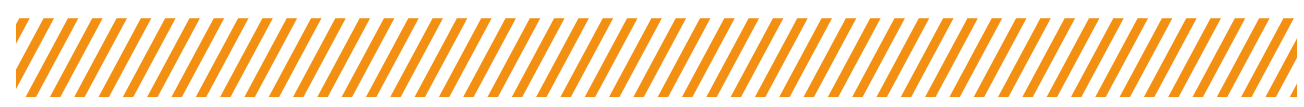

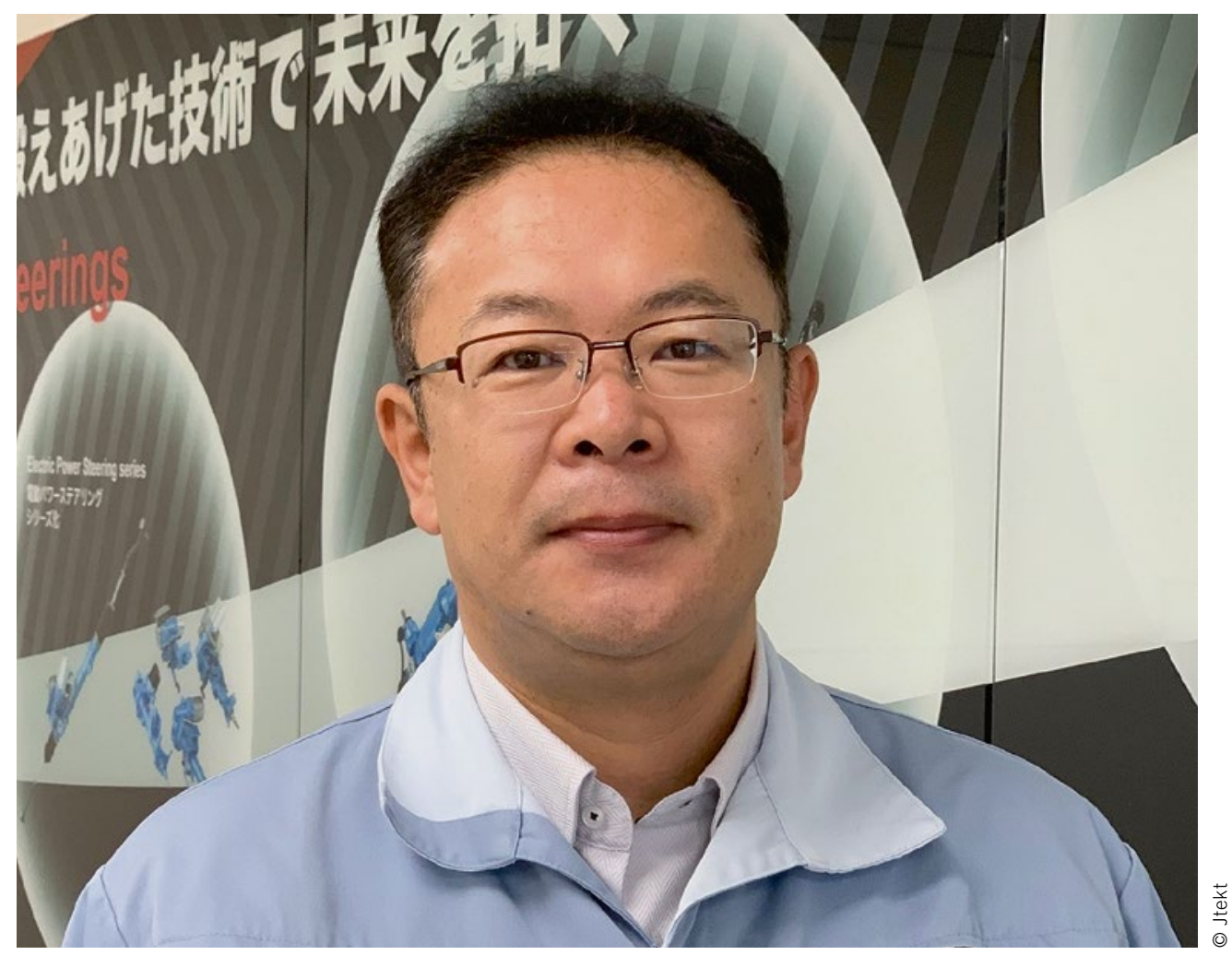

Yasuyuki Yoshii, B. Eng.

General Manager System Innovation

Research Department at Jtekt

\section{Harmonizing Driver and Automation}

After having underestimated the immense effort required to put highly automated driving cars on the market, the world's first launch of a hands-off single-lane driving capabilities vehicle has been reached with the Nissan Skyline (ProPILOT 2.0) in 2019. 2020 will be remembered as a milestone for the automotive industry with many OEMs introducing vehicles with an automation SAE level around 3. In Japan, this was planned to be highlighted at the now postponed 2020 Summer Olympics in Tokyo. Japan already used the Tokyo Olympics 1964 to successfully launch a new technology: the Shinkansen, the world's first high-speed train with an unprecedented record of punctuality and no fatalities. The debate on whether or not to skip SAE level 3 is cut short by accomplished facts. The essential point is the fact that today's technology does not yet permit to take the driver out of the equation. Automation is a trend motivated by promises of better traffic safety and reduction of driver workload. Airplanes and automobiles are the two means of transport where this trend is relevant. Nevertheless, most of the latest commercial airplane crashes and SAE level 2 car accidents are in some way related to poor communication between the operator and the machine, confusion arising from the process of regaining manual control and misuse. Human-machine interaction is becoming a fundamental issue to be addressed so as to guarantee that automation will effectively deliver on its promises.

Highly automated vehicles are often seen as having two distinctive operating modes: manual and automated. This suggests that one precludes the other. With the automation system active, the driver is thought to be unable to control the vehicle. This dual-mode model raises the problem of transitioning between modes as a singular event that should be executed with a great deal of care and attention to the limitations of automation and driver readiness. At Jtekt research headquarters, we have opted for the less constraining approach of sharing lateral control of the vehicle between the driver and the vehicle. Haptic methods are used as a means of harmonizing the driver and the vehicle through the steering system by enabling manual inputs without deactivation of the automation in an intuitive manner. The vehicle control can be adjusted within the whole range of operation from manual to fully automated driving depending on the driver and traffic conditions. Transition timing becomes irrelevant, and the driver does not need to reengage the automated mode after each manual operation. But the quest for harmonizing driver and automation goes even further by reconsidering how the vehicle interior can reflect these operational changes. 